\title{
Establishment of a novel mouse model of adenomyosis suitable for longitudinal and quantitative analysis and perinatal outcome studies
}

Mohammed Elsherbini

The University of Tokyo

Kaori Koga ( $\nabla$ kawotan-tky@umin.ac.jp )

The University of Tokyo

Takehiro Hiraoka

The University of Tokyo

Keiichi Kumasawa

The University of Tokyo

\section{Erina Satake}

The University of Tokyo

Ayumi Taguchi

The University of Tokyo

\section{Tomoko Makabe}

The University of Tokyo

\section{Arisa Takeuchi}

The University of Tokyo

Gentaro Izumi

The University of Tokyo

Masashi Takamura

Saitama Medical University

Miyuki Harada

The University of Tokyo

\section{Tetsuya Hirata}

Doai Kinen Hospital

\section{Yasushi Hirota}

The University of Tokyo

Osamu Wada-Hiraike

The University of Tokyo

\section{Yutaka Osuga}

The University of Tokyo 


\section{Research Article}

Keywords: Adenomyosis, animal model, CD31, cell proliferation, fibrosis, Ki67, Masson's trichrome staining, puncture, pregnancy, vascular density

Posted Date: March 1st, 2022

DOI: https://doi.org/10.21203/rs.3.rs-1332354/v2

License: (c) (i) This work is licensed under a Creative Commons Attribution 4.0 International License.

Read Full License

Version of Record: A version of this preprint was published at Scientific Reports on October 20th, 2022. See the published version at https://doi.org/10.1038/s41598-022-22413-8. 


\section{Abstract}

Background: The purpose of this study was to establish a novel mouse model of adenomyosis suitable for longitudinal and quantitative analyses and perinatal outcome studies.

Methods: Using a $30 \mathrm{G}$ needle, the entire uterine wall of one horn was mechanically punctured at a frequency of 100 times $/ 1 \mathrm{~cm}$ (adenomyosis horn). The other horn was left unpunctured (control horn). Mice were sacrificed on day 14 (D14) or day 65 (D65). The uterus was fixed, paraffin-embedded, sliced, and stained. Lesions were detected and counted, and their volumes were measured. Cell proliferation and fibrosis were assessed by Ki67 and Masson's Trichrome staining, respectively. Blood vessels were detected using CD31 immunostaining. Some of the mice were mated, and the date of delivery, litter size, number of implantations, and number and volume of postpartum lesions were measured.

Results: The number of lesions per horn did not differ between D14 and D65. The volume of the entire lesion was significantly greater on D65 than on D14 ( $<<0.0001)$. The volume of the epithelial part of the lesion was significantly greater in D65 ( $p<0.0001)$. The volume of the stromal part of the lesion was also greater on D65 ( $<$ 0.0001). The percentage of Ki67 positive cells in the epithelial part of the lesion was significantly higher on D14 ( $<0.05)$. In contrast, the percentage of Ki67-positive cells in the stromal part was significantly higher on D65 ( $p<0.01)$. Vascular density in the lesions was higher in on D65 $(p<$ $0.05)$. The percentage of fibrotic area was significantly higher on D65 $(p<0.01)$. The date of delivery was slightly earlier than that reported for healthy mice of the same strain. The litter size was smaller than that reported in previous research. The number of implantation sites did not differ between the control and the adenomyosis horn. The number and volume of lesions did not differ between the non-pregnant and postpartum groups.

Conclusions: This model can be applied to evaluate the pathogenesis of adenomyosis, validate the efficacy of therapeutic agents, and evaluate the effect of adenomyosis on pregnancy and vice versa.

\section{Background}

Adenomyosis is a benign gynecological condition characterized by the presence of endometrium-like epithelial and stromal tissues in the myometrium. Despite the prevalence and severity of symptoms such as pain and heavy menstrual bleeding (HMB), its pathogenesis and etiology have not yet been elucidated [1-4]. It has been proposed that the cause of adenomyosis is invasion of the endometrium into the myometrium due to mechanical damage to the endometrium during delivery or intrauterine surgery [1] [5]; however, there has been no experimental validation of this theory.

Recently, with the development of noninvasive diagnostic methods such as MRI, adenomyosis can now be diagnosed without a hysterectomy, and it has been revealed that this disease can cause pain, HMB, and infertility [2] [6-8]. Patients with adenomyosis have also been found to have an increased risk of adverse perinatal events such as preterm delivery, fetal growth restriction, and preeclampsia [9-12]. The 
mechanisms by which adenomyosis causes perinatal adverse events have not yet been clarified, and no experimental methods have been established to verify them.

Animal models are useful in the study of adenomyosis to elucidate its pathogenesis and to develop therapies. To date, several laboratories have established mouse models of adenomyosis $[13,14][15-19]$. However, most models are not suitable for longitudinal studies because of the long time between lesion induction and establishment and the lack of knowledge regarding long-term lesion maintenance [20]. In addition, many endocrinologically induced adenomyosis models, such as the pituitary isograft mouse model [13], prolonged progesterone exposure [14] and prolonged tamoxifen exposure [15-19] are not suitable for pregnancy studies because they require hormonal modification or oophorectomy.

The purpose of this study was to establish a novel mouse model of adenomyosis that was suitable for longitudinal and quantitative analyses and perinatal outcome studies. To create this model, we attempted to mimic the mechanical damage of the endometrium, which is generally considered a main cause of adenomyosis, in the mouse uterus.

\section{Methods}

\section{Induction of adenomyosis by mechanical stimulation of the mouse uterus}

All procedures described in this study were conducted in accordance with the guidelines and regulations of the Animal Care and Use of the University of Tokyo Committee. Six-week-old BALB/c female mice were purchased from Japan SLC, Inc. (Tokyo, Japan). The mice were fed a mouse diet and water and maintained on a light/dark cycle ( $12 \mathrm{~h} / 12 \mathrm{~h}$ ) under controlled living conditions. The adenomyosis mouse model was established as follows. First, a midline incision was made in the abdomen to expose the uterine horns. Using a $30 \mathrm{G}(1 / 2 \mathrm{inch})$ needle, the entire uterine wall of one horn was mechanically punctured at a frequency of 100 times $/ 1 \mathrm{~cm}$ (adenomyosis horn). The other horn was left unpunctured (control horn). The puncture penetrated through the entire uterine wall, as shown in Fig. 1A. The mice were sacrificed 14 (D14) and 65 days (D65) after the operation, and uteri were collected ( $n=3$ for each timepoint).

\section{Tissue preparation for hematoxylin and eosin staining (H\&E staining)}

Uterine tissues were sectioned, fixed in $10 \%$ formalin, dehydrated gradually in ethanol, and embedded in paraffin at $60^{\circ} \mathrm{C}$. For hematoxylin and eosin staining (H\&E staining), sections were cut horizontally at a thickness of $6 \mu \mathrm{m}$ and mounted on slides for analysis.

\section{Immunohistochemistry (IHC)}


We investigated the extent of the adenomyosis lesions by cutting through the entire horn and quantitatively analyzing the number and volume of lesions. To confirm the presence of lesions, we prepared serial sections and performed immunohistochemistry. One section was stained with cytokeratin 8 (CK8) to detect epithelial cells and the other section with alpha smooth muscle actin (aSMA) to detect smooth muscle cells. When epithelial cells were found within a smooth muscle layer, the area was considered an adenomyosis lesion. IHC was conducted as follows. All sections were deparaffinized in xylene and hydrated in a series of graded alcohols $(100 \%, 100 \%, 100 \%, 90 \%, 80 \%$, and $70 \%)$. Antigen retrieval was performed by boiling the samples in an ethylenediaminetetraacetic acid (EDTA) antigen retrieval solution (pH 9) (DAKO, Cat. No. K8004, Glostrup, Denmark) for $45 \mathrm{~min}$ at $98^{\circ} \mathrm{C}$. The samples were then incubated at room temperature for $30 \mathrm{~min}$. The samples were washed three times in phosphatebuffered saline (PBS) and incubated with a peroxidase blocking agent (DAKO, Cat. No. S2023) for 10 min. The samples were washed again and incubated overnight with the diluted primary antibodies listed in Table 1 at $4^{\circ} \mathrm{C}$. The samples were then washed with PBS and incubated with the secondary antibody from the REAL EnVision Detection System (DAKO, Cat. No. K5007) at room temperature for 30 min. Afterward, the samples were visualized using 3,3'-

diaminobenzidine (DAB) chromogen from the REAL EnVision Detection System (DAKO, Cat. No. K5007). Samples were counterstained with hematoxylin, gradually dehydrated using graded alcohol and xylene, and then mounted with mounting medium.

Table 1

The list of the primary antibodies for immunohistochemistry

\begin{tabular}{|llll|}
\hline Molecule & Dilution & Origin & Supplier (catalog no,) \\
\hline CK8 & $1 / 1000(\mathrm{IHC})$ & Rabbit monoclonal (EP1628Y) & ABCAM (Cat. \#ab53280) \\
& $1 / 100(\mathrm{IF})$ & & \\
\hline Alpha SMA & $1 / 1000(\mathrm{IHC})$ & Rabbit monoclonal (EPR5368) & ABCAM (Cat. \#ab124964) \\
\hline Alpha SMA & $1 / 100(\mathrm{IF})$ & Goat polyclonal & ABCAM (Cat. \#ab21027) \\
\hline Ki67 & $1 / 500(\mathrm{IHC})$ & Rabbit monoclonal (Clone SP6) & Funakoshi (Cat. \#RM-9106-S1) \\
\hline CD31 & $1 / 300(\mathrm{IHC})$ & Rabbit monoclonal (D8V9E) & Cell Signaling (Cat. \#77699) \\
\hline
\end{tabular}

\section{Immunofluorescent staining}

Paraffin-embedded samples were sliced at a thickness of $5 \mu \mathrm{m}$, deparaffinized in xylene, and rehydrated in a graded alcohol sequence. Antigen retrieval was performed as previously described. Sections were then co-incubated with rabbit anti-CK8 and goat anti-aSMA antibodies (Table 1). Immunofluorescence detection was performed using secondary Alexa Fluor 488 goat anti-rabbit IgG $(H+L)$ pAb (1:200; A11034; RRID: AB_2576217; Thermo Fisher Scientific, Waltham, MA) and secondary Alexa 568 donkey anti-goat IgG $(H+L)$ pAb (1:200; A-11057; RRID: AB_2534104; Thermo Fisher Scientific) before incubation for $120 \mathrm{~min}$ at room temperature. The samples were counterstained with 4',6-diamidino-2-phenylindole 
(DAPI). Fluorescent images were obtained using a Zeiss LSM 700 confocal microscope (Carl Zeiss, Germany).

\section{Measurement of the number and volume of the adenomyosis lesions}

This study developed a novel method to quantitatively analyze the number and volume of adenomyosis lesions. Once the uterine horn was obtained, the tissues were fixed and embedded in paraffin blocks. The entire uterus was sliced into 6- $\mu \mathrm{m}$-thick serial sections. On average, 2500 sections were prepared from one horn. All H\&E-stained sections were observed, and the number of adenomyosis lesions was counted. The length was calculated by multiplying the total number of slices containing a single lesion (from top to bottom) by $6 \mu \mathrm{m}$. The cross-sectional area of the lesion was measured using ImageJ (version 1.53c, National Institutes of Health), by tracing the innermost line of the smooth muscle that was identified by positive staining with aSMA and using it as the outermost line of the lesion (Fig. 2A). The cross-sectional area of the epithelial part of the lesion was measured by identifying regions of epithelium within the adenomyosis lesion with positive CK8 staining (Fig. 2A). The cross-sectional area of the stromal part of the lesion was calculated by subtracting the area of the epithelial part from the area of the entire lesion. The volumes of the lesions were calculated as the sum of the cross-sectional areas multiplied by the inter-slide spacing. The equation used to calculate this is as follows:

Volume $\left(\mathrm{mm}^{3}\right)=\sum$ cross-sectional area $\left(\mathrm{mm}^{2}\right) \times 0.006(\mathrm{~mm})$.

\section{Evaluation of cell proliferation}

Cell proliferation was evaluated by Ki67 IHC. Cells with Ki67-positive nuclei in the epithelial and stromal parts of adenomyosis lesions were counted, and the percentage of cells that were Ki67-positive was calculated.

\section{Evaluation of vascular density}

For vascular density evaluation, CD31, a marker for endothelial cells, was used to detect blood vessels. Blood vessels within the lesions were identified, the cross-sectional area of blood vessels was measured, and this was divided by the cross-sectional area of the adenomyosis lesion to calculate the vascular density.

\section{Evaluation of fibrosis}

Fibrotic regions were identified using Masson's trichrome staining. The uterine sections were deparaffinized, rehydrated, and transferred to Bouin's solution at $56^{\circ} \mathrm{C}$ for $15 \mathrm{~min}$. Sections were stained using Masson's Trichrome Staining kit (Sigma-Aldrich Inc., St. Louis, MO) following the manufacturer's instructions. Images of adenomyosis lesions were randomly taken at a magnification of $40 x$. The crosssectional area of fibrotic regions (areas where collagen deposition had occurred) was determined using image analysis software ImageJ (version 1.53c, National Institutes of Health, Bethesda, MD) with the plugin "color deconvolution" for stain separation (MTS1,2). Regions that stained bluer than the 
automatically calculated threshold were identified as fibrotic, and the cross-sectional areas of these regions were calculated. The percentage of the lesion that was fibrotic was calculated by dividing the area of the fibrotic region by the area of the entire lesion.

\section{Establishment and evaluation of mouse pregnancy model}

To investigate the prognosis of pregnancy in this mouse model and whether the lesions persisted after pregnancy, seven mice were used to model pregnancy. Six-week-old male BALB/c mice were used for mating. Mating was started 7 days after the establishment of adenomyosis and continued for up to 10 consecutive days until the plug was confirmed. Female mice were then kept in a separate cage until delivery, and the date of delivery was recorded. The pups were separated from the dams on the first day after delivery, and litter size was recorded. The dams were sacrificed on the third day after delivery, and the uteri were collected. The number of implantation sites in both horns was determined and recorded. The number and volume of adenomyosis lesions were evaluated as described above and compared with those of non-pregnant mice.

\section{Statistical analysis}

All data analysis were conducted using JMP Pro 15 software (SAS Institute Inc., Cary, NC). All nonparametric data were compared using Wilcoxon rank sum test. $p<0.05$ was considered significant.

\section{Results}

\section{Establishment of mechanical induced adenomyosis mouse model}

For all mice, one horn was punctured, which was defined as the adenomyosis horn while the other horn was left intact, which was defined as the control horn. The mean time from the start of anesthesia to the end of procedure was approximately 20 minutes (data not shown). There were no postoperative complications such as infection or adhesion.

Figure 1a A schema showing the punctures of uterine horn The entire uterine wall of one horn was mechanically punctured at a frequency of 100 times $/ 1 \mathrm{~cm}$. The puncture was crossed the whole uterine wall thickness. b A macroscopic finding of the uterus (D65) Adenomyosis horn was shorter than the control horn. $c$ A representative microscopic image of the control horn $(D 65, \times 100)$ Hematoxylin staining (HE) shows the normal uterine architecture; cytokeratin 8 (CK8) shows eutopic endometrium and alpha smooth muscle actin (a SMA) shows smooth muscle architecture. $d$ A representative microscopic image of the adenomyosis horn (D65, upper line; $\times 100)$ (lower line; $\times 400)$ Hematoxylin staining (HE) shows the uterine structure with an adenomyosis lesion (black box); cytokeratin 8 (CK8) shows eutopic endometrial epithelium and epithelium of the lesion (marked with black box), alpha smooth muscle actin (a SMA) shows smooth muscle structure that encircles adenomyosis lesion (marked with black box). 


\section{Changes in the number, size of adenomyosis lesions over time}

Figure 2a A representative immunofluorescence image of the adenomyosis lesion $(\times 400)$ at the 14 th postoperative day (D14) (upper side) and the 65th postoperative day (D65) (lower side) with cytokeratin 8 (CK8) (red) and alpha smooth muscle actin (a SMA) (green). In the illustration, the area highlighted in green $(E)$ is the area of the epithelium of the adenomyosis lesion stained with CK8, and the area highlighted in gray $(S)$ is the area of stroma of the lesion, which is not stained with either aSMA or CK8. $\mathbf{b}$ A scatter plot for the adenomyosis lesion count per horn Lesion count per horn was compared between the D14 and D65. The count was not significantly different between the groups. c A box and whisker graph for the adenomyosis lesion volume The lesion volume of the entire, epithelial, and stromal area was compared between D14 and D65. The cross-sectional area of the lesion was measured by tracing the innermost line of the smooth muscle that was identified by positive staining with aSMA and using it as the outermost line of the lesion. The cross-sectional area of the epithelial part of the lesion was measured by tracing the epithelium, with positive CK8 staining, within the adenomyosis lesion. The cross-sectional area of the stromal part of the lesion was calculated by subtracting the area of the epithelial part from the area of the entire lesion. The volumes of each lesion were calculated as the sum of cross-sectional areas multiplied by the inter-slide spacing. The volume was significantly larger in D65 than in D14 $(p<0.0001)$. Box: $25-75 \%$, Whisker: $10-90 \%$, Midline: median, NS: not significantly different $* *$ : $p<0.0001$.

\section{Changes in cell proliferation, vascular density, and fibrosis of adenomyosis lesions over time}

As shown in Fig. 3A, the percentage of Ki67 positive cells in epithelial part of the lesion was significantly higher in D14 than in D65 (D14: $15.3 \pm 5.0 \%, D 65: 2.6 \pm 1.3 \%, p<0.05$ ), while in the stromal part, the percentage of Ki67 positive cells was significantly higher in D65 than in D14 (D14: $0.5 \pm 0.4 \%$, D65: $6.3 \pm$ $1.6 \%, \mathrm{p}<0.01$ ). As for vascular density (Fig. 3B) the density was significantly higher in D65 than in D14 (D14: $0.06 \pm 0.01 \%, D 65: 0.1 \pm 0.01 \%, p<0.05$ ). The percentage of fibrotic area was significantly higher in D65 than in D14 (D14: $0.007 \pm 0.004 \%$, D65: $5.1 \pm 1.01, p<0.01$ ) (Fig. 3C).

\section{Evaluation pregnancy outcome and postpartum lesion in adenomyosis model mice}

Five out of seven mice $(71.4 \%)$ conceived within 10 days of consecutive mating. The date of delivery, the litter size, and the number of implantation sites of each mouse are shown in Table 2 . The date of delivery was $19.2 \pm 0.8$ (mean \pm SD) dpc which is slightly earlier than previously reported for the mouse of the same strain (mean $\pm S D ; 20.2 \pm 0.1 \mathrm{dpc}$ ) [21] and (mean \pm SD; $19.6 \pm 0.6 \mathrm{dpc}$ ) [22]. The litter size was $4.4 \pm$ 3.4 (mean \pm SD), which is smaller than litter size that previously reported in two articles $(5.9 \pm 2.7$ [22] and $5.32 \pm 0.2$ [23]). The number of implantation sites were not different between the control and the adenomyosis horn (control: $2.6 \pm 1.8$, adenomyosis: $3 \pm 1.6$, mean $\pm S D, p=0.7$ ) (Table 2). 
Table 2

Adenomyosis pregnancy model

\begin{tabular}{|c|c|c|c|c|}
\hline \multirow[t]{2}{*}{ Mouse } & \multirow{2}{*}{$\begin{array}{l}\text { Delivery day } \\
\text { (dpc) }\end{array}$} & \multirow{2}{*}{$\begin{array}{l}\text { Litter size } \\
\text { (No.) }\end{array}$} & \multicolumn{2}{|c|}{ Implantation site } \\
\hline & & & $\begin{array}{l}\text { Control } \\
\text { horn }\end{array}$ & $\begin{array}{l}\text { Adenomyosis } \\
\text { horn }\end{array}$ \\
\hline A & 20 & 2 & 1 & 1 \\
\hline B & 19 & 9 & 5 & 4 \\
\hline C & 18 & 7 & 4 & 5 \\
\hline D & 20 & 3 & 2 & 3 \\
\hline E & 19 & 1 & 1 & 2 \\
\hline Mean \pm SD & $19.2 \pm 0.8$ & $4.4 \pm 3.4$ & $2.6 \pm 1.8$ & $3 \pm 1.6$ \\
\hline
\end{tabular}

We then evaluated the number and the volume of the adenomyosis lesions in the postpartum mouse and compared them with those in non-pregnant mouse. The lesion count per horn was not different between the groups (non-pregnant: $18.6 \pm 2.7$, postpartum: $20.5 \pm 2.3$, mean $\pm S E M, p=0.6$, Fig. $4 A$ ). The volume of the entire lesion was also comparable between the groups (non-pregnant: $109.6 \pm 16.5$, postpartum: 89.1 $\pm 18.8 \mathrm{~mm}^{3} /$ lesion, $p=0.6$, Fig. 4B left). The volume of the epithelial part of the lesion was also not different between the groups (non-pregnant: $45.1 \pm 7.1$, postpartum group: $31.7 \pm 6.9 \mathrm{~mm}^{3} /$ lesion, $p=0.2$, Fig. 4B middle). The volume of the stromal part of the lesion was also not different between the groups (non-pregnant: $64.5 \pm 10$, postpartum: $57.5 \pm 12.4 \mathrm{~mm}^{3} /$ lesion, $p=0.9$, Fig. 4B right).

\section{Discussion}

In this study, we established a novel mouse model of adenomyosis induced by direct mechanical puncture of the uterus. The established lesions had glandular tubular structures consisting of epithelium and stroma within the myometrium, similar to lesions seen in human adenomyosis. We also quantitatively assessed the changes in these lesions over time and found that the lesions decreased in number and increased in size. We also found that, over a 2-month period, epithelial cell proliferation decreased, stromal cell proliferation increased, and vascular density and fibrosis increased. In addition, we found the mice were capable of pregnancy and delivery, and that their lesions persisted after delivery.

This novel adenomyosis model was established by mechanically disrupting the endometrial-myometrial interface. As mentioned in the introduction, several theories have been proposed to explain the etiology of adenomyosis. At least one type of adenomyosis (Type 1 adenomyosis in Kishi's classification) is known to be more prevalent in women of multiparity and women who have undergone uterine surgery [24-26]. It is widely believed that this type of adenomyosis is caused by mechanical damage to the uterus that disrupts the endometrial-myometrial interface and allows the endometrium to invade the myometrium 
[19] [24] [27, 28]. In the present mouse model, we attempted to disrupt the endometrial-myometrial interface with repeated punctures that penetrated the entire myometrium. Using this method, we succeeded in generating glandular structures consisting of epithelium and stroma in the myometrium, which resembled human adenomyosis.

One of the advantages of this model is that following uterine puncture, which was a short procedure, the lesion persisted for a long time. Previously described mouse models of adenomyosis take significantly longer to establish. In previous studies, lesions have only been established 10-12 weeks after intervention [20] or 6 weeks after neonatal tamoxifen administration [15]. In contrast, in our model, lesions are established by day 7 at the latest, which has the advantage of allowing us to study drug administration and pregnancy prognosis early after uterine puncture, while the mice are young. Furthermore, this model was shown to create lesions that persisted for at least 65 days. In contrast, previous studies on mouse models did not examine whether the lesions persisted for a long time, except for a single study by Shen et al. [17] where lesions were examined on day 60 . Thus, this model could be useful to confirm the efficacy of long-term drug treatment.

In the current study, we also established a novel quantitative analysis method for adenomyosis lesions, which involved counting and measuring the volume of lesions using slices of the entire horn.

Furthermore, by using markers for epithelial cells and myocytes, we established a method to measure and evaluate the epithelial and stromal parts of the lesion independently. This quantitative analysis allowed for a better understanding of temporal changes in lesion growth and regression. The present study revealed that, over time, the number of lesions significantly decreased, and the volume of each lesion significantly increased in both the epithelial and stromal parts. However, it is unclear whether this was due to the fusion of multiple lesions or the regression of some lesions and the growth of others. This is an area that requires further study.

Following on from the previously described finding regarding the increase in lesion size over time, the current study also examined the changes in cell proliferation for both epithelial and stromal compartments over time using Ki67 staining. We found that, in the epithelial compartment, Ki67-positive cells were significantly more abundant in the early stage. In contrast, Ki67-positive cells became significantly more abundant in the stromal compartment as the lesion progressed. This suggests that, in adenomyosis lesions, epithelial cells proliferate first and stromal cells proliferate later, but it is still unclear whether this is due to; epithelial-mesenchymal transition (EMT) or because the epithelial cells cause the attraction and the proliferation of stromal cells by some unknown mechanism. Cell proliferation in eutopic and ectopic endometrium has been studied in endometriosis [29, 30], but not in adenomyosis; therefore, further studies using human specimens and comparisons with the current study should be conducted in the future.

In addition to quantitative assessment, we also qualitatively assessed the characteristics of the lesions, including vessel density and fibrosis. Regarding the vascular density, it has been demonstrated that microvessels develop around adenomyosis lesions in human adenomyosis [31, 32]. In the present study, 
the vascular density progressively and significantly increased over time, which is consistent with findings made in studies using other mouse models of adenomyosis $[17,18]$. It is possible that the proliferation of these microvessels was responsible for the increase in the volume of adenomyosis lesions over time in our model. It is well known that human adenomyosis lesions are accompanied by fibrosis [33] due to repeated cyclic bleeding associated with tissue injury and repair [34, 35]. In our model, the extent of fibrosis in the adenomyotic lesion significantly increased over time, a finding that is consistent with discoveries made in other studies [17]. The gross findings that the adenomyosis horns were shorter than the control horns may also be due to the contraction of the muscle layer caused by fibrosis. In summary, this model allows quantitative observation of angiogenesis and fibrosis associated with adenomyosis over time and is expected to be useful in determining the efficacy of treatment methods in the future.

Furthermore, we confirmed that these mice were able to conceive and deliver, and that the lesions persisted after delivery. To the best of our knowledge, there has been only one report [19] that studied the course of pregnancy using an adenomyosis mouse model, but that report was limited to examining litter size by sacrificing dams on their fourth day of pregnancy. Therefore, the current study is the first study in which mice were allowed to deliver in order to analyze perinatal outcomes and the status of adenomyosis after delivery. One advantage of our model over the previous model is that it does not require hormonal modification or oophorectomy, which can cause ovarian dysfunction or uterine thinning [13-19] and may affect the course of pregnancy. This study showed that the number and size of adenomyosis lesions did not change between the pre-pregnancy and post-partum period; therefore, this model may be useful for modelling perinatal outcomes in pregnancies complicated by adenomyosis.

Finally, our preliminary data on perinatal outcomes showed that adenomyosis mice seemed to have an earlier delivery date and smaller litter size than normal healthy mice of the same strain and age, as reported in previous studies [21-23]. We also found that the number of implantation sites was comparable between the adenomyosis horn and the control horn, suggesting that the presence of adenomyosis not only affects implantation but also affects intrauterine development. These outcomes seem to mimic the high incidence of preterm birth, fetal growth restriction (FGR) and intrauterine fetal death (IUFD).in pregnant women with adenomyosis [9-12], and this mouse model is expected to be a suitable model of pregnancy complicated by adenomyosis, although further validation is warranted.

\section{Conclusions}

In this study, we established a mouse model of adenomyosis caused by mechanical damage to the uterus, and showed that the number and volume of lesions could be longitudinally and quantitatively analyzed. Furthermore, we proved that this model can conceive, deliver, and persist after delivery. Collectively, this model can be applied to investigate the pathogenesis of adenomyosis, validate the efficacy of therapeutic agents, and evaluate the effect of adenomyosis on pregnancy and vice versa.

\section{Abbreviations}


aSMA: alpha smooth muscle actin; CK8: Cytokeratin 8; D14: Day 14; D65: Day 65; DAPI: 4, 6-diamidino-2phenylindole; H\&E: hematoxylin and eosin; HMB: heavy menstrual bleeding; IHC: Immunohistochemistry, EMT; epithelial-mesenchymal transition, FGR; fetal growth restriction, IUFD; intrauterine fetal death.

\section{Declarations}

\section{Ethics approval}

All animal experiments were approved by the Animal Committee of The University of Tokyo School of Medicine (Approval No.P19-034, 11 July 2019), and the manuscript follows the recommendations of the ARRIVE guidelines.

\section{Consent to Publish}

Not applicable

\section{Availability of data and materials}

The datasets used and/or analysed during the current study are available from the corresponding author on reasonable request.

\section{Competing Interests}

The authors declare that they have no competing interests

\section{Funding}

This work was supported by grants from the Japan Agency for Medical Research, JSPS KAKENHI and Society of Women's Health Science Research.

\section{Authors' contributions}

ME, EM, TH and AT performed mouse experiments. ME, KK, ES, AT, TM, GI and TH acquired, analyzed and interpreted the data. $\mathrm{ME}$ and $\mathrm{KK}$ drafted the manuscript. $\mathrm{MT}, \mathrm{MH}, \mathrm{YH}, \mathrm{OWH}$ and $\mathrm{YO}$ critically and substantially revised the manuscript. All authors read and approved the final manuscript.

\section{Acknowledgements}

The authors thank Mrs. Megumi Tobita for her technical assistants.

\section{References}

1. Garcia-Solares J, Donnez J, Donnez O, Dolmans MM: Pathogenesis of uterine adenomyosis: invagination or metaplasia? Fertil Steril 2018, 109(3):371-379. 
2. Devlieger R, D'Hooghe T, Timmerman D: Uterine adenomyosis in the infertility clinic. Hum Reprod Update 2003, 9(2):139-147.

3. Neriishi K, Hirata T, Fukuda S, Izumi G, Nakazawa A, Yamamoto N, Harada M, Hirota Y, Koga K, WadaHiraike $O$ et al: Long-term dienogest administration in patients with symptomatic adenomyosis. $J$ Obstet Gynaecol Res 2018, 44(8):1439-1444.

4. Takamura M, Koga K, Harada M, Hirota Y, Fujii T, Osuga Y: A case of hemorrhagic shock occurred during dienogest therapy for uterine adenomyosis. J Obstet Gynaecol Res 2020.

5. Leyendecker G, Wildt L, Mall G: The pathophysiology of endometriosis and adenomyosis: tissue injury and repair. Arch Gynecol Obstet 2009, 280(4):529-538.

6. Harada T, Khine YM, Kaponis A, Nikellis T, Decavalas G, Taniguchi F: The Impact of Adenomyosis on Women's Fertility. Obstet Gynecol Surv 2016, 71(9):557-568.

7. Soave I, Wenger JM, Pluchino N, Marci R: Treatment options and reproductive outcome for adenomyosis-associated infertility. Curr Med Res Opin 2018, 34(5):839-849.

8. Sharma S, Bathwal S, Agarwal N, Chattopadhyay R, Saha I, Chakravarty B: Does presence of adenomyosis affect reproductive outcome in IVF cycles? A retrospective analysis of 973 patients. Reprod Biomed Online 2019, 38(1):13-21.

9. Hashimoto A, Iriyama T, Sayama S, Nakayama T, Komatsu A, Miyauchi A, Nishii O, Nagamatsu T, Osuga Y, Fujii T: Adenomyosis and adverse perinatal outcomes: increased risk of second trimester miscarriage, preeclampsia, and placental malposition. J Matern Fetal Neonatal Med 2018, 31(3):364-369.

10. Harada T, Taniguchi F, Amano H, Kurozawa Y, Ideno Y, Hayashi K, Harada T, Japan E, Children's Study G: Adverse obstetrical outcomes for women with endometriosis and adenomyosis: A large cohort of the Japan Environment and Children's Study. PLoS One 2019, 14(8):e0220256.

11. Liu XY, Zhang Y, Wei Y, Li R, Zhao YY: [Perinatal outcome of pregnant women with adenomyosis]. Zhonghua Fu Chan Ke Za Zhi 2020, 55(11):743-748.

12. Nirgianakis K, Kalaitzopoulos DR, Schwartz ASK, Spaanderman M, Kramer BW, Mueller MD, Mueller M: Fertility, pregnancy and neonatal outcomes of patients with adenomyosis: a systematic review and meta-analysis. Reprod Biomed Online 2021, 42(1):185-206.

13. Huseby RA, Soares MJ, Talamantes F: Ectopic pituitary grafts in mice: hormone levels, effects on fertility, and the development of adenomyosis uteri, prolactinomas, and mammary carcinomas. Endocrinology 1985, 116(4):1440-1448.

14. Lipschutz A, Iglesias R, Panasevich VI, Salinas S: Pathological changes induced in the uterus of mice with the prolonged administration of progesterone and 19-nor-contraceptives. British Journal of Cancer 1967, 21(1):160-165.

15. Green AR, Styles JA, Parrott EL, Gray D, Edwards RE, Smith AG, Gant TW, Greaves P, Al-Azzawi F, White IN: Neonatal tamoxifen treatment of mice leads to adenomyosis but not uterine cancer. Exp Toxicol Pathol 2005, 56(4-5):255-263. 
16. Mehasseb MK, Bell SC, Habiba MA: The effects of tamoxifen and estradiol on myometrial differentiation and organization during early uterine development in the CD1 mouse. Reproduction 2009, 138(2):341-350.

17. Shen M, Liu X, Zhang H, Guo SW: Transforming growth factor beta1 signaling coincides with epithelial-mesenchymal transition and fibroblast-to-myofibroblast transdifferentiation in the development of adenomyosis in mice. Hum Reprod 2016, 31(2):355-369.

18. Jin Z, Wu X, Liu H, Xu C: Celecoxib, a selective COX-2 inhibitor, markedly reduced the severity of tamoxifen-induced adenomyosis in a murine model. Exp Ther Med 2020, 19(5):3289-3299.

19. Guo S, Lu X, Gu R, Zhang D, Sun Y, Feng Y: Transcriptome analysis of endometrial tissues following GnRH agonist treatment in a mouse adenomyosis model. Drug Des Devel Ther 2017, 11:695-704.

20. Hao M, Liu X, Guo SW: Adenomyosis in mice resulting from mechanically or thermally induced endometrial-myometrial interface disruption and its possible prevention. Reprod Biomed Online 2020, 41(5):925-942.

21. Zhang WS, Xie QS, Wu XH, Liang QH: Neuromedin B and its receptor induce labor onset and are associated with the RELA (NFKB P65)/IL6 pathway in pregnant mice. Biol Reprod 2011, 84(1):113117.

22. Finlay JB, Liu X, Ermel RW, Adamson TW: Maternal Weight Gain as a Predictor of Litter Size in Swiss Webster, C57BL/6J, and BALB/cJ mice. J Am Assoc Lab Anim Sci 2015, 54(6):694-699.

23. Moreira VB, Mattaraia VGM, Moura ASAMT: Lifetime reproductive efficiency of BALB/c mouse pairs after an environmental modification at 3 mating ages. Journal of the American Association for Laboratory Animal Science: JAALAS 2015, 54(1):29-34.

24. Kishi $Y$, Suginami $H$, Kuramori R, Yabuta $M$, Suginami R, Taniguchi F: Four subtypes of adenomyosis assessed by magnetic resonance imaging and their specification. Am J Obstet Gynecol 2012, 207(2):114 e111-117.

25. Taran FA, Weaver AL, Coddington CC, Stewart EA: Understanding adenomyosis: a case control study. Fertil Steril 2010, 94(4):1223-1228.

26. Panganamamula UR, Harmanli OH, Isik-Akbay EF, Grotegut CA, Dandolu V, Gaughan JP: Is prior uterine surgery a risk factor for adenomyosis? Obstet Gynecol 2004, 104(5 Pt 1):1034-1038.

27. Donnez J, Donnez O, Dolmans MM: Introduction: Uterine adenomyosis, another enigmatic disease of our time. Fertil Steril 2018, 109(3):369-370.

28. Uduwela AS, Perera MA, Aiqing L, Fraser IS: Endometrial-myometrial interface: relationship to adenomyosis and changes in pregnancy. Obstet Gynecol Surv 2000, 55(6):390-400.

29. Franco-Murillo Y, Miranda-Rodriguez JA, Rendon-Huerta E, Montano LF, Cornejo GV, Gomez LP, Valdez-Morales FJ, Gonzalez-Sanchez I, Cerbon M: Unremitting cell proliferation in the secretory phase of eutopic endometriosis: involvement of pAkt and pGSK3beta. Reprod Sci 2015, 22(4):502510.

30. Colón-Caraballo M, García M, Mendoza A, Flores I: Human Endometriosis Tissue Microarray Reveals Site-specific Expression of Estrogen Receptors, Progesterone Receptor, and Ki67. Appl 
Immunohistochem Mol Morphol 2019, 27(7):491-500.

31. Schindl M, Birner P, Obermair A, Kiesel L, Wenzl R: Increased microvessel density in adenomyosis uteri. Fertil Steril 2001, 75(1):131-135.

32. Nie J, Liu X, Zheng Y, Geng JG, Guo SW: Increased immunoreactivity to SLIT/ROBO1 and its correlation with severity of dysmenorrhea in adenomyosis. Fertil Steril 2011, 95(3):1164-1167.

33. Kobayashi H, Kishi Y, Matsubara S: Mechanisms Underlying Adenomyosis-Related Fibrogenesis. Gynecol Obstet Invest 2020, 85(1):1-12.

34. Hinz B: Formation and function of the myofibroblast during tissue repair. J Invest Dermatol 2007, 127(3):526-537.

35. Thannickal VJ, Zhou Y, Gaggar A, Duncan SR: Fibrosis: ultimate and proximate causes. J Clin Invest 2014, 124(11):4673-4677.

\section{Figures}


A

Control Adenomyosis

horn horn

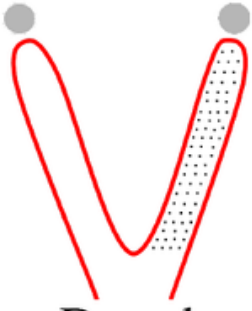

Dorsal

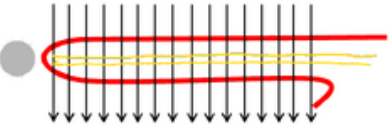

Sagittal

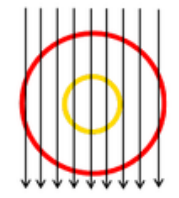

Transverse
B

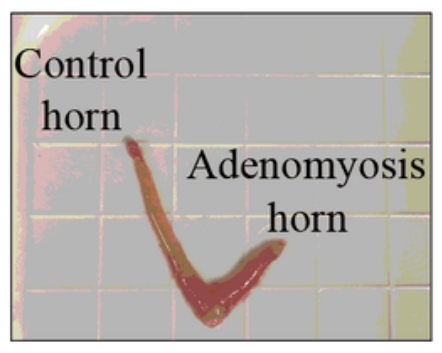

C

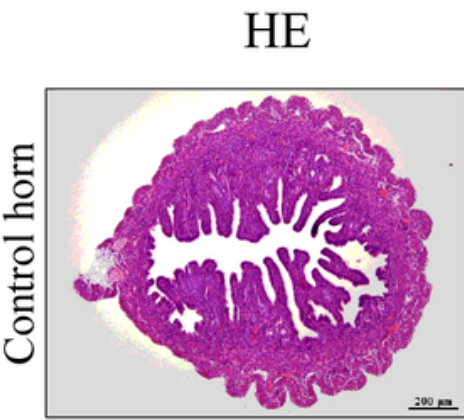

CK8
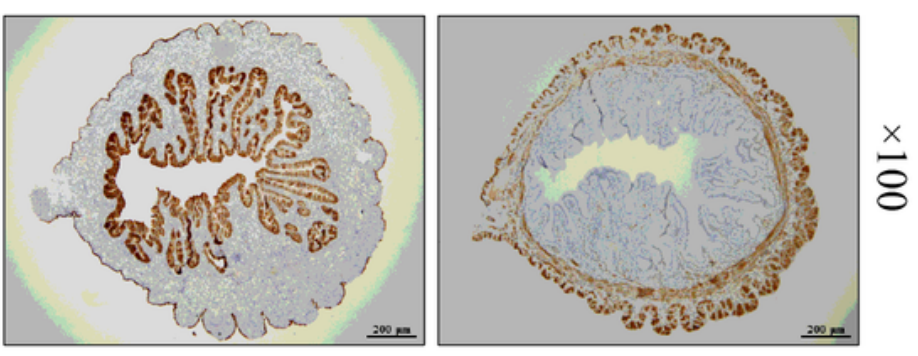

D

HE

CK8

aSMA
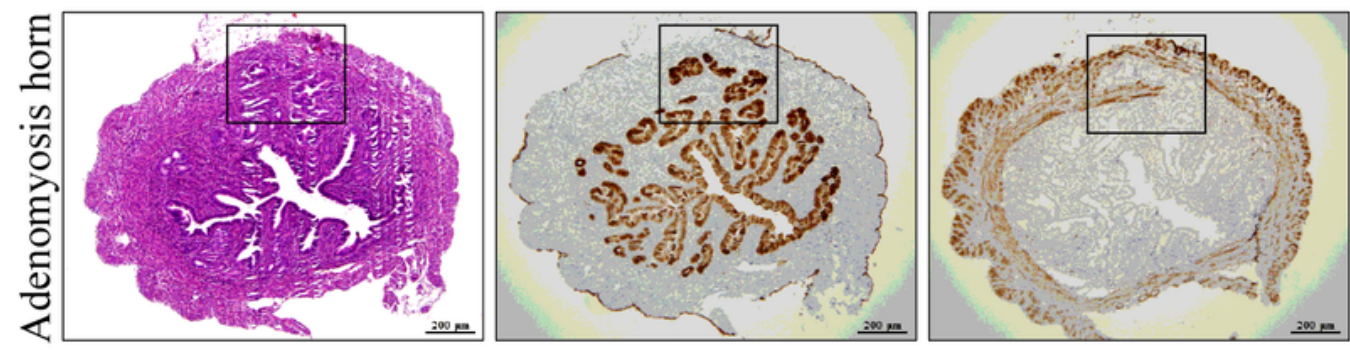

$\stackrel{x}{8}$
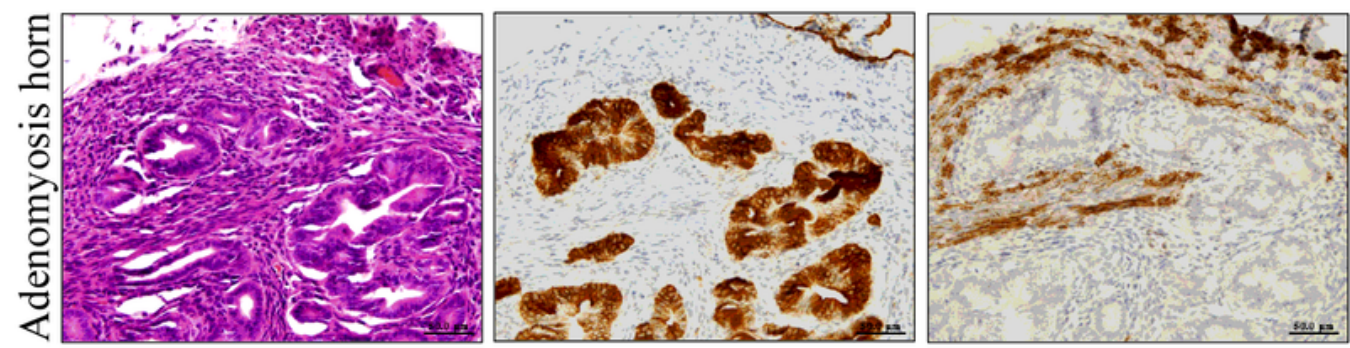

敦

Figure 1

a A schema showing the punctures of uterine horn The entire uterine wall of one horn was mechanically punctured at a frequency of 100 times $/ 1 \mathrm{~cm}$. The puncture was crossed the whole uterine wall thickness. b A macroscopic finding of the uterus (D65) Adenomyosis horn was shorter than the control horn. c A representative microscopic image of the control horn $(D 65, \times 100)$ Hematoxylin staining $(\mathrm{HE})$ shows the normal uterine architecture; cytokeratin 8 (CK8) shows eutopic endometrium and alpha smooth muscle 
actin (a SMA) shows smooth muscle architecture. $\mathbf{d}$ A representative microscopic image of the adenomyosis horn (D65, upper line; $\times 100)$ (lower line; $\times 400)$ Hematoxylin staining (HE) shows the uterine structure with an adenomyosis lesion (black box); cytokeratin 8 (CK8) shows eutopic endometrial epithelium and epithelium of the lesion (marked with black box), alpha smooth muscle actin (a SMA) shows smooth muscle structure that encircles adenomyosis lesion (marked with black box).

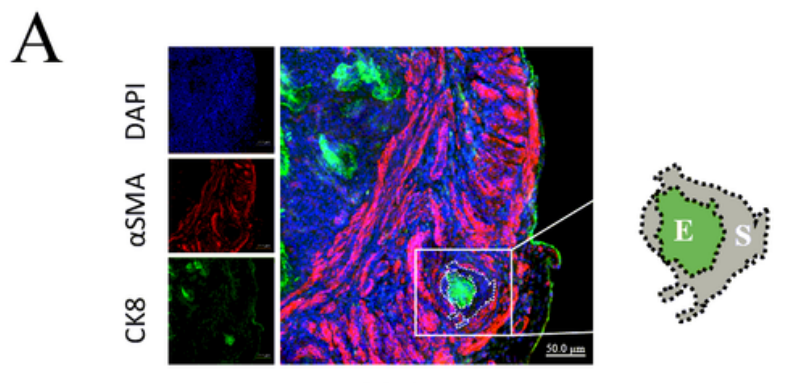

D14

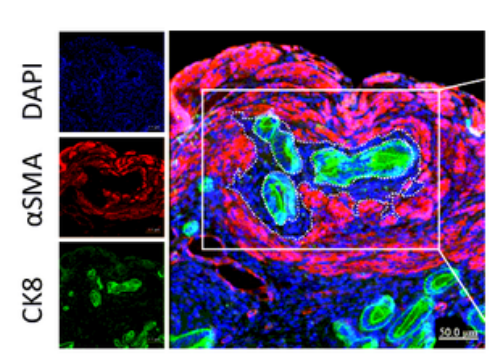

D65

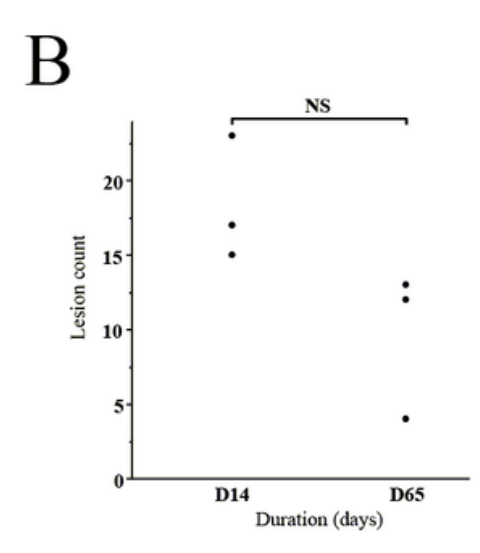

Lesion count/horn

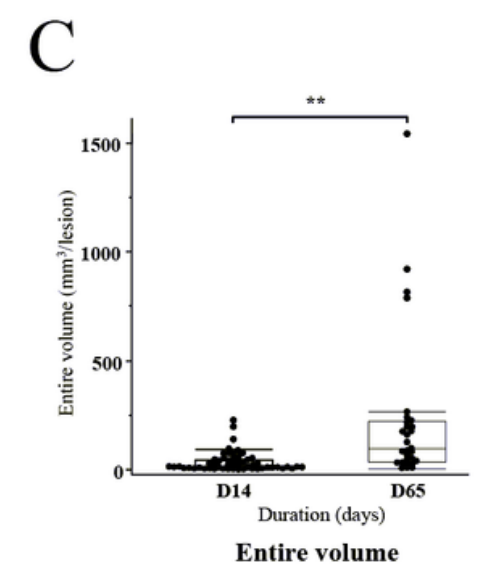

Entire volume

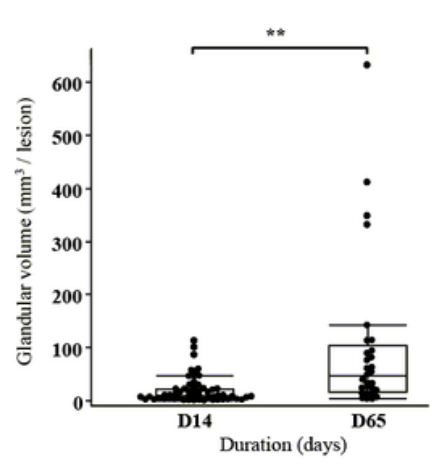

Epithelial volume

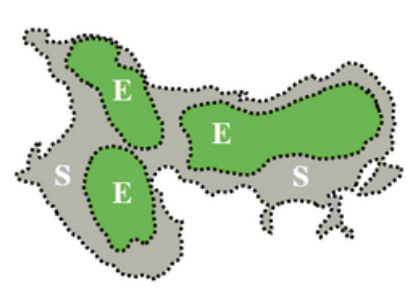

(1) 
of cross-sectional areas multiplied by the inter-slide spacing.The volume was significantly larger in D65 than in D14 ( $<$ 0.0001). Box: 25-75\%, Whisker: 10-90\%, Midline: median, NS: not significantly different $\star *: p<0.0001$.

A

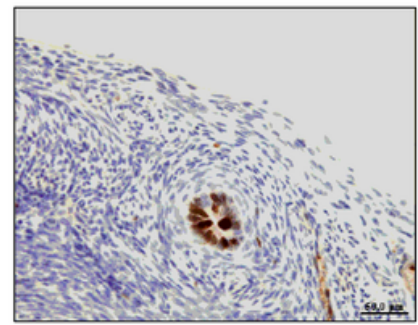

D14
Ki67 expression

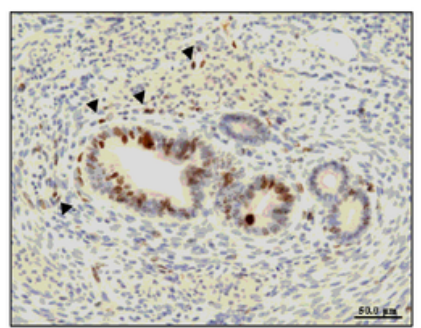

D65

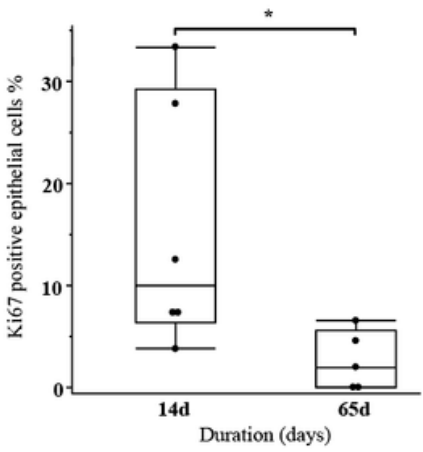

Ki67 positive epithelial cells

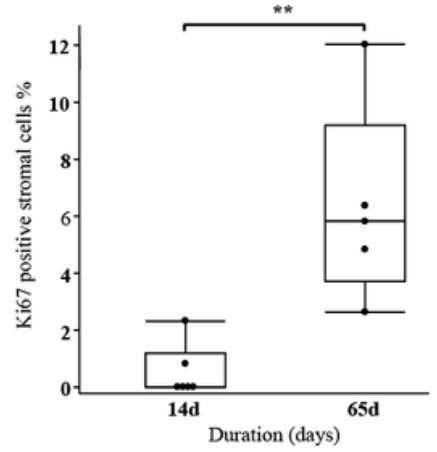

Ki67 positive stromal cells

$\mathrm{B}$

CD31 expression

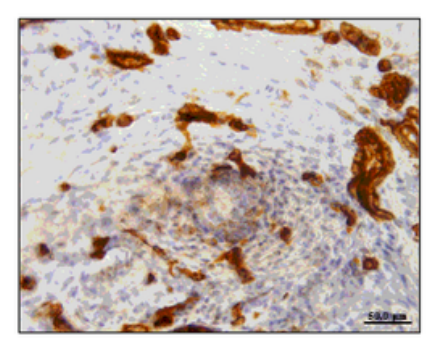

D14

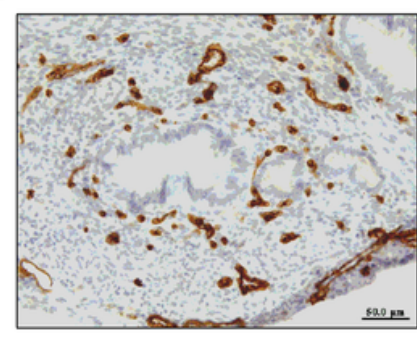

D65

C

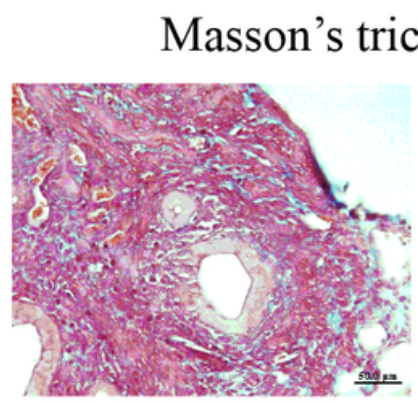

D14

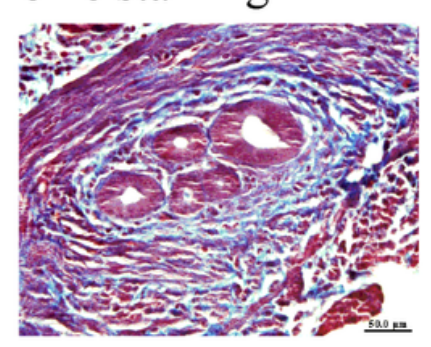

D65
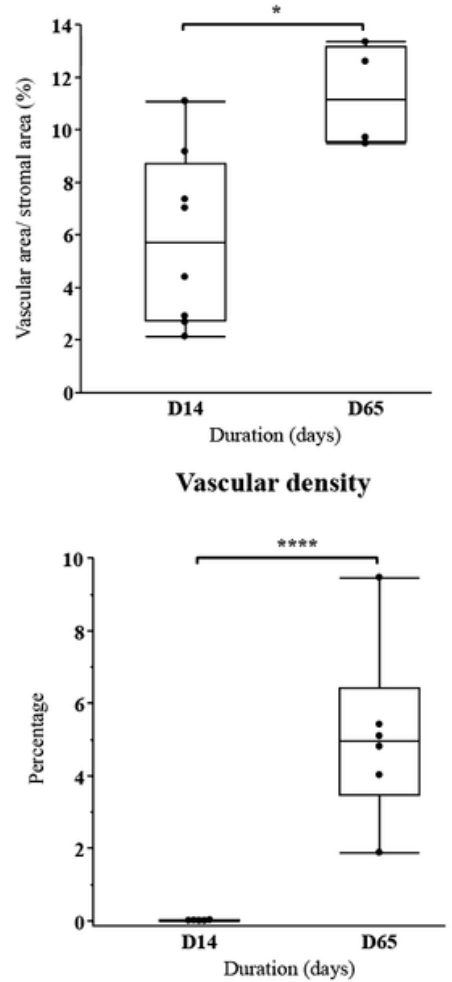

Proportion of fibrosis

Figure 3

a A representative microscopic image of Ki67 expression in adenomyosis lesion $(\times 400)$ and a box and whisker graph of the percentage of Ki67 positive cells Ki67 is positive in the nuclei of both epithelial and stromal cells (arrowheads) in the adenomyosis lesion at the $14^{\text {th }}$ (D14) (left) and the $65^{\text {th }}$ (D65) (right) postoperative day. In epithelial cells, the percentage of Ki67 positive cells was significantly higher in D14 than in D65 ( $p<0.05)$, while in stromal cells, the percentage was significantly higher in D65 than in D14 $(p<0.01)$. b A representative microscopic image of CD31 expression in adenomyosis lesion $(\times 400)$ and a 
box and whisker graph of vascular density CD31, a marker for endothelial cells, was used to detect blood vessels. Blood vessels within the lesion were identified, the cross-sectional area of blood vessels was measured, and this was divided by the cross-sectional area of adenomyosis lesion to calculate the vascular density. Vascular density in D65 was significantly higher than that in D14 $(p<0.05)$. c A representative microscopic image of Masson's trichrome staining of adenomyosis lesion $(\times 400)$ and a box and whisker graph of percentage of fibrotic area Areas of fibrosis (collagen deposition) were stained blue. The percentage of fibrosis area in D65 was significantly higher than that in D14 $(p<0.001)$. Box: 25-75\%, Whisker: 10-90\%, Midline: median, $*$ : $<<0.05, * \star: p<0.01 .,{ }^{\star \star * \star}: p<0.001$.

A

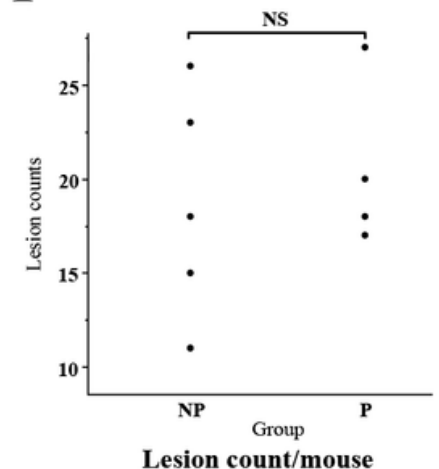

$\mathrm{B}$

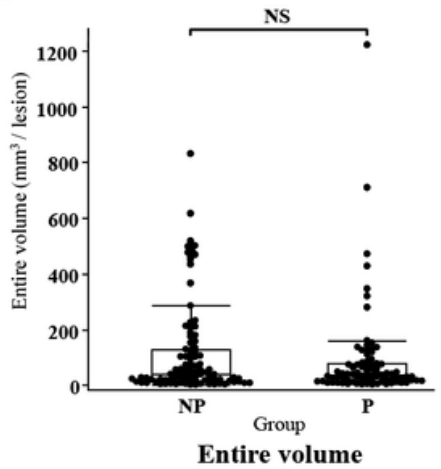

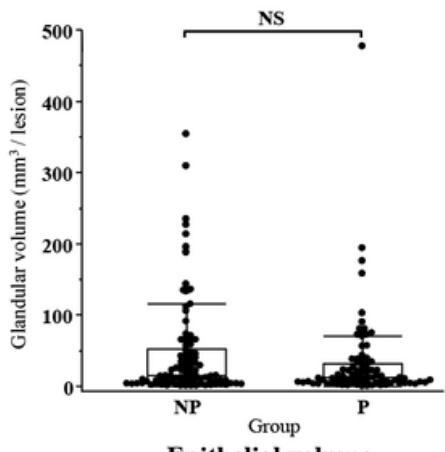

Epithelial volume

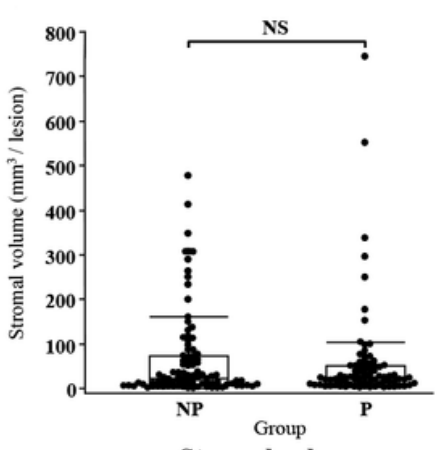

Stromal volume

Figure 4

a A scatter plot for adenomyosis lesion count per each mouse The lesion count in non-pregnant (NP) and postpartum $(\mathrm{P})$ mice were compared. $\mathbf{b}$ A box and whisker graph for adenomyosis lesion volume The cross-sectional area of the lesion was measured by tracing the innermost line of the smooth muscle that was identified by positive staining with aSMA and using it as the outermost line of the lesion. The crosssectional area of the epithelial part of the lesion was measured by tracing the epithelium, with positive CK8 staining, within the adenomyosis lesion. The cross-sectional area of the stromal part of the lesion was calculated by subtracting the area of the epithelial part from the area of the entire lesion. The volumes of each lesion were calculated as the sum of cross-sectional areas multiplied by the inter-slide spacing. The lesion volume of the entire, epithelial, and stromal area was compared between NP and $P$. The lesion counts and lesion volume of the entire, epithelial and stromal area was comparable between the group. Box: $25-75 \%$, Whisker: 10-90\%, Midline: median, NS: $p>0.05$. 